\title{
Erfahrungen mit dem neuen Bibliothekssystem aDIS/BMS - Teil 2
}

aDIS/BMS löst BIBDIA in der Leihstelle ab

\section{Vorarbeiten und erfolgreicher Start}

Nachdem das System BIBDIA 13 Jahre in der Leihstelle der WLB im Einsatz gewesen war, wurde es am 11.09.2013 gegen 21:00 Uhr abgestellt. Für die Mitarbeiterinnen und Mitarbeiter der Leihstelle galt es, sich von einem zuverlässigen, stabilen und sehr vertrauten Ausleihsystem zu verabschieden.

\section{Ein Rückblick im Zeitraffer}

Im Februar 2013 stand uns zum ersten Mal das Ausleihgrundfenster von aDIS/BMS mit Fremddaten zur Verfügung, und mit großer Spannung und in freudiger Erwartung begannen wir, uns mit den Ausleihfunktionen des neuen Systems zu beschäftigen, zunächst durch Ausprobieren, Testen und Sammeln von Fragen - auf gut deutsch „trial and error".

Für die Migration beschrieben wir die Ausgangsdaten in BIBDIA und beschäftigten uns mit den möglichen Zuordnungen im neuen System. Konkordanzen zu allen Feldern mussten gefunden werden. Wir entwickelten neue Benutzergruppen in aDIS/BMS, in denen die Ausleih- und Gebührengruppen von BIBDIA zusammengefasst werden mussten. Für die Migration der Exemplardaten von BIBDIA nach aDIS/BMS wurden umfangreiche Excel-Tabellen erstellt.

BIBDIA-Medientypen, -Entleihbarkeiten, -Standorte (z.B. Hauptmagazin oder Magazin Fellbach), -Kommentare (z.B. die verschiedenen Farben der Supplementbestände bei Periodika), -Infodaten (verschiedene Kennzeichen die Info-Datei betreffend) wurden in entsprechenden aDIS/BMSMedientypcodes (MTCs) zusammengefasst. Diese steuern die Bestell- und Entleihbarkeit von Medien und sind somit eine wichtige Grundlage für das neue Ausleihsystem bzw. den OPAC. Unsere Vorschläge wurden mit denen der Medienbearbeitung diskutiert, und gemeinsam erstellten wir diese elementare Tabelle für die Migration. Der Wunsch, Alt- und Sonderbestände künftig online bestellbar zu machen, wurde mit den Kolleginnen und Kollegen des Hauptlesesaals und der Sonderlesesäle abgestimmt und umgesetzt. Um eine gute Zusammenführung und Aufbereitung unserer Daten zu gewährleisten, waren wir während der gesamten Vorbereitungsphase damit befasst, zusammen mit der IT-Abteilung fehlerhafte Einträge in den BIBDIADatenbanken zu recherchieren, zu korrigieren oder zu löschen.

Wegen des engen Zeitplans des Migrationsprojektes, und da die Firma aStec sich zu diesem Zeitpunkt (April) noch intensiv mit anderen Bibliotheksumstellungen beschäftigen musste (u.a. mit der UB Freiburg, die kurz vor uns umstellte), blieb für unsere Anfragen und Probleme zum System so gut wie keine Zeit übrig.

Daher besuchten wir die Kolleginnen in der BLB Karlsruhe und der UB Tübingen, um zum einen aDIS/BMS in der Ausleihpraxis zu sehen und zum anderen verschiedene Fragen und Probleme zu klären. Diese Besuche waren immer äußerst hilfreich und beruhigend. Dafür möchten wir uns an dieser Stelle nochmals herzlich bei den Kolleginnen bedanken.

Im Juni stand uns die 1. Testmigration mit den eigenen Benutzerdaten und den bis dahin von uns festgelegten Konditionen zur Verfügung, und sowohl der Test-Dienstclient als auch der Test-OPAC konnte von allen Stammkräften der Leihstelle geprüft und getestet werden. Bereits zu diesem Zeitpunkt war klar, dass der ursprünglich für den 12.08.2013 vorgesehene Go-live-Termin verschoben werden musste (zunächst auf 10.09.2013 und schlussendlich auf 16.09.2013).

Während der Testphase fand auch die Multiplikatorenschulung der Firma für die Projektgruppe Ausleihe statt. Auf deren Basis erstellten wir detaillierte Schulungsunterlagen und begannen Anfang August mit den Schulungen der Stamm- und Aushilfskräfte der Leihstelle (leider nicht wie geplant mit der 
2. Testmigration, sondern mit der fehlerhaften 1. Testmigration). Auch die Kolleginnen und Kollegen, die samstags die Leihstelle unterstützen, wurden eingelernt.

Parallel dazu wurden für alle Benutzergruppen die endgültigen Benutzungskonditionen festgelegt (immer in Bezug auf die definierten Medientypen), insgesamt für 20 Benutzergruppen und 31 MTCs. Auf diesen Festlegungen basieren Bestellungen, Ausleihverbuchungen, Vormerkungen, Gebührenberechnungen und Sperren.

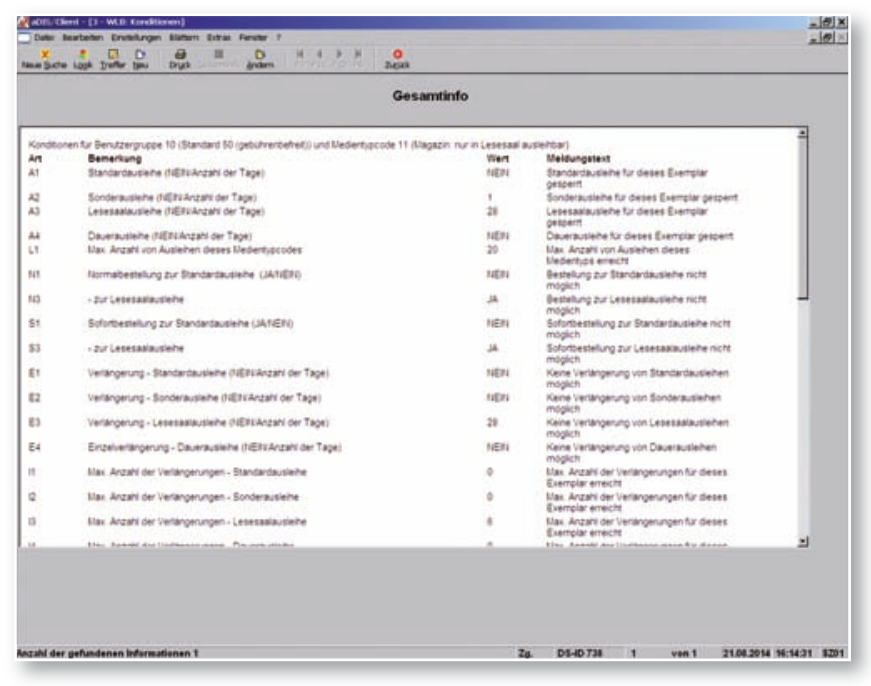

Abb. 1: Auszug aus der Tabelle einer Benutzungskondition

Beschäftigungsrechte für die Kolleginnen und Kollegen wurden vergeben, Fangschaltungen definiert, Listboxelemente bestimmt, Homepageänderungen und Infoblätter für die Benutzer vorbereitet, um an dieser Stelle nur die wichtigsten Tätigkeiten zu nennen.

Der ohnehin ambitionierte Zeitplan wurde immer enger. Die 2. Testmigration wurde erst am 26.08.2013 zum Prüfen freigegeben. Trotz vieler „Baustellen“ und sehr verkürzter Testzeit konnte der dritte avisierte Go-live-Termin nicht nochmals verschoben werden. Am 04.09.2013 stand der genaue Zeitplan für die Umstellung endgültig fest. Die Benutzer wurden im Internet und mittels Flyer genau über die Serviceeinschränkungen durch den anstehenden Systemwechsel informiert. Alle Beteiligten arbeiteten auf Hochtouren. Täglich schickten wir Dutzende von E-Mails hin und her und trafen letzte Entscheidungen.

Dann war es soweit: Am 16.09.2013 gab unsere IT-Abteilung aDIS/BMS intern für die Leihstelle frei. Wir begannen mit dem Abarbeiten der angesammelten Rückbuchungen, Verlängerungen etc., die während der Serviceeinschränkungen nicht durchgeführt werden konnten. Am 17.09.2013 ab 9:00 Uhr wurden die Schalter und der Selbstabholerbereich geöffnet und ab 12:00 Uhr der OPAC für Bestellungen freigegeben. Der erste mit Spannung erwartete Magazinbestellzetteldruck mit aDIS/BMS erfolgte um 13:00 Uhr.

Fazit nach dem 1. Öffnungstag mit aDIS/BMS: Die Umstellung war geglückt! Die intensiven Vorbereitungen haben sich ausgezahlt. Alle Kolleginnen und Kollegen der Leihstelle waren bestens vorbereitet und konnten somit dem ersten Benutzeransturm mit dem neuen System souverän standhalten.

\section{Integriertes Bibliothekssystem im Alltag}

Das Ausleihgrundfenster des neuen Systems ist anwenderfreundlich und übersichtlich. Es zeigt alle wichtigen Informationen auf einen Blick.

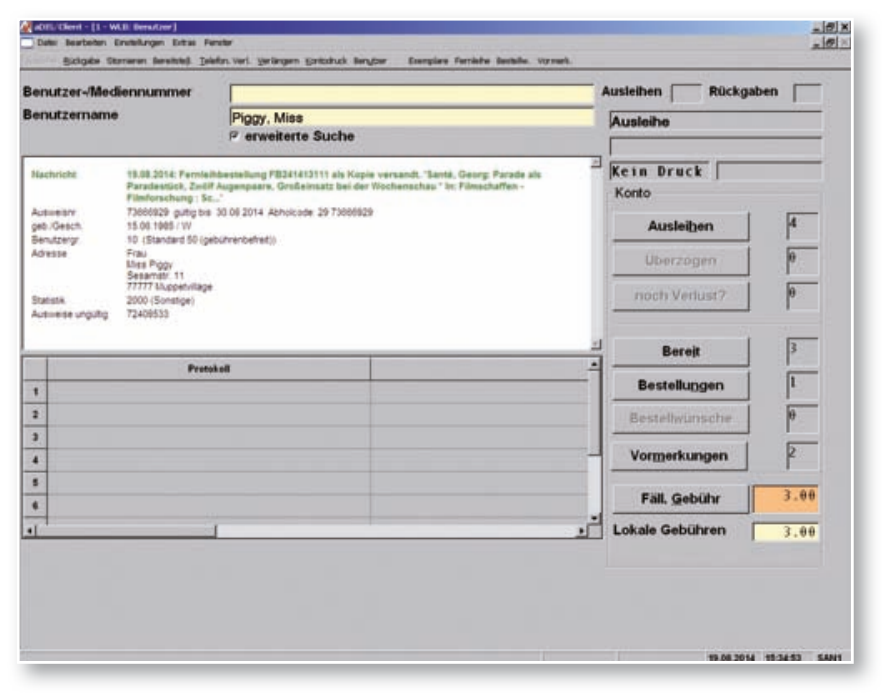

Abb. 2: Das Ausleihgrundfenster von aDIS/BMS mit den wichtigsten Informationen

Bisher mussten Ausleihfunktionen mittels bestimmter Kennziffern per Tastatur aufgerufen werden, bei aDIS/BMS, einem Windows-kompatiblen System, können viele Thekentätigkeiten mit Maus oder Tastatur und Shortcuts (Hotkeys) effizient durchgeführt werden.

Der große Vorteil des integrierten Bibliothekssystems: Alle Medien werden mit Titeln aufgeführt (ausgenommen noch nicht konvertierter Bestand). 


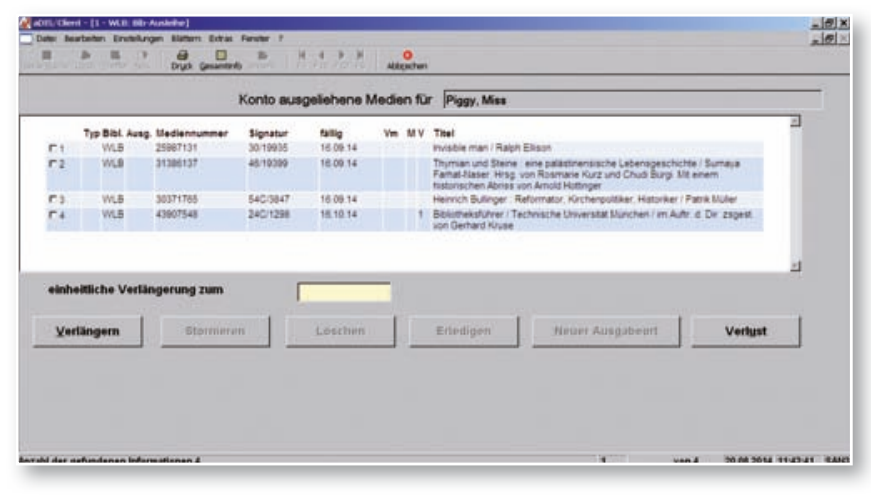

Abb. 3: Kontoauszug der ausgeliehenen Medien eines Benutzers

\section{Interne Neuerungen}

- Alle Mitarbeiterinnen und Mitarbeiter können Bestellfunktionen nur noch über den OPAC aufrufen.

- Für alle Mitarbeiterkonten (dienstliche und private) wurde die „automatische Verlängerung" eingerichtet, maximales Ausleihfenster ist ein Jahr.

- Dienst- und Geschäftsgangsbestellungen gibt es in aDIS/BMS nicht mehr. Daher wurden für Abteilungen - sofern nicht bereits vorhanden Dienstkonten eingerichtet und auf Wunsch Arbeitskonten für Kolleginnen und Kollegen.

- Die Verbuchung von Medien, die in Ausstellungen oder Semesterapparate kommen, musste neu konzipiert werden. Damit die Bücher auf diese Konten verbucht werden können und nicht vormerkbar sind, mussten dafür eigene Aufstellungen und Standorte eingerichtet werden.

- Die Sacherschließungskonten konnten aufgelöst werden, da die Sacherschließung in den Geschäftsgang integriert wurde.

- Das Abräumen im Selbstabholerbereich erfolgt anhand einer Abräumliste, die auch Stornierungen durch die Benutzer berücksichtigt.

- Neu ist auch das Feld "Nachricht". Es bietet uns die Möglichkeit, dem Benutzer schnell und unkompliziert eine Nachricht zukommen zu lassen, die er in seinem OPAC-Konto einsehen kann.

- Das Freibuchen, das in aDIS/BMS das frühere Absignieren ablöst, ist durch das integrierte Bibliothekssystem erheblich komplexer und differenzierter geworden.
Bei der Zusammenführung der Exemplardaten der drei verschiedenen Systeme (Verbund, BIBDIA und Libero) sind zwangsläufig Probleme entstanden ${ }^{1}$, die auf verschiedene Arten gelöst werden mussten. Diese Lösungen müssen beim Freibuchen berücksichtigt werden, und deshalb sind oft aufwändige Nacharbeiten bei den Exemplaren nötig. Da das Rekonversionsprojekt ${ }^{2}$ noch nicht abgeschlossen ist, muss für die Benutzer die "freie Signaturenbestellung" zugelassen werden. Auch dies erschwert das Freibuchen. Ungenaue, unvollständige Magazinbestellungen müssen von den Mitarbeiterinnen und Mitarbeitern des Magazins und der Leihstelle oftmals im OPAC nachrecherchiert und ergänzt werden, da nur so positive Erledigungen der Bestellungen möglich sind (vor allem bei Zeitschriftenbestellungen). Beim Freibuchen findet gleichzeitig die RFID-Konvertierung statt.

\section{Neuerungen für Benutzer}

Über ihr Konto im OPAC (,Mein Konto“) können Benutzer ihre offenen Bestellungen nicht nur einsehen, sondern auch jederzeit löschen. Die bereitgestellten Medien können ebenso storniert werden.

Die Grundleihfrist von 28 Tagen bei der Ausleihe haben wir beibehalten. Bei den Verlängerungen wurde das "Mannheimer Modell“ übernommen, d.h. in einem Ausleihfenster von maximal 112 Tagen (4 x 28 Tage) sind Verlängerungen jeden Tag möglich.

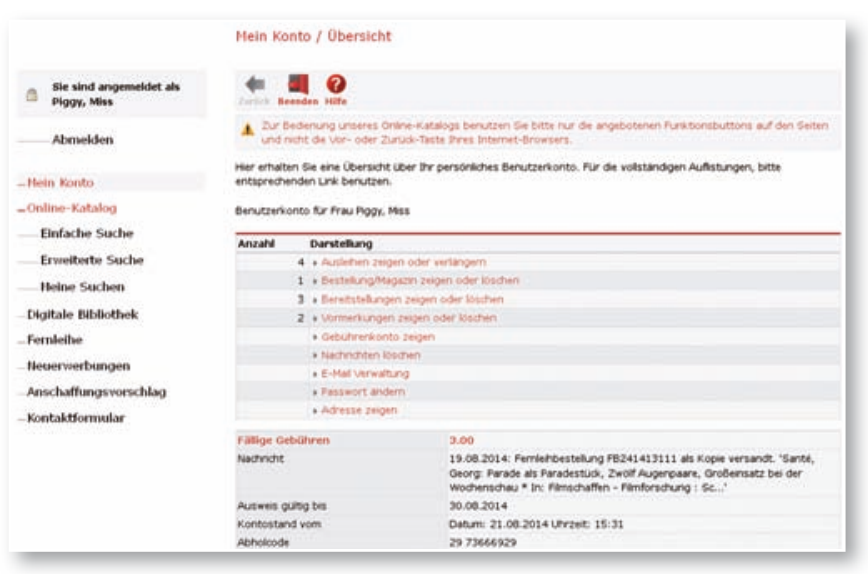

Abb. 4: Übersicht über ein Benutzerkonto im OPAC

Neu ist ebenso, dass sich Benutzer auf neu erworbene Medien, die noch im Geschäftsgang sind, vormerken können. Auch auf von der Medienbearbeitung erst bestellte Medien kann bereits eine Vormerkung eingetragen werden. 
Neben Abholbenachrichtigungen und Voraberinnerungen werden nun auch Mahnschreiben für die gebührenpflichtigen 1. bis 3. Mahnungen sowie Widerrufe der Leihfristverlängerungen bei Fernleihentleihungen per E-Mail verschickt. Benutzer, die keine E-Mail-Adresse hinterlegt haben, werden jetzt per Brief (davor per Postkarte) benachrichtigt und müssen dadurch höhere Portogebühren bezahlen. aDIS/BMS erlaubt den Benutzern, Magazinbestellungen in Auftrag zu geben, auch wenn die Gültigkeit des Bibliotheksausweises bereits abgelaufen ist. Die Mahnintervalle wurden vereinheitlicht: Grundsätzlich setzt das Mahnverfahren am dritten Tag nach Ablauf der Leihfrist ein.

Eine der Stärken von aDIS/BMS ist die erprobte RFID-Verarbeitung. Daher konnten wir bereits kurze Zeit nach Umstellungsbeginn die wohl spektakulärste Neuerung für die Benutzer (und auch für uns) einführen: die Selbstverbuchung ${ }^{3}$.

\section{Fazit und Ausblick}

Nach beinahe einem Jahr Arbeiten mit dem Ausleihmodul von aDIS/BMS lässt sich sagen, es ist ein gut funktionierendes, flexibles und belastbares System, mit dem wir sehr schnell vertraut wurden und das schnell erlernbar ist.
Ein Ausleihsystem kann jedoch nur gut funktionieren, wenn ein guter OPAC dahinter steht, denn als reine Magazinbibliothek sind wir auf exakte Magazinbestellungen angewiesen.

aDIS/BMS ist ein sehr komplexes System und bietet zweifelsohne viele Möglichkeiten - wir lernen täglich dazu. Aber nicht alle vorhandenen Einstellungen entsprechen unseren organisatorischen und strukturellen Bedingungen und können somit nicht ohne Änderungen eingesetzt werden, z.B. die Verlustbearbeitung, die Gebührenanmahnungen und die verkürzten Leihfristen bei Vormerkungen. Wünschenswert wäre auch, dass auf den Kontoauszügen vorgemerkte Medien erkennbar sind. Noch müssen Geschäftsgänge überdacht und geändert werden. Besonders bei abteilungsübergreifenden Arbeitsabläufen sind Anpassungen und Weiterentwicklungen nötig. Aber da die Projektgruppen schon in der stressigen und heftigen Vorbereitungszeit intensiv und konstruktiv zusammengearbeitet und sich immer gut verstanden haben, freuen wir uns auch darauf.

Willkommen Zukunft!

Eva Jantsch

Marion Wildermuth

\section{Soziale Netzwerke}

\section{- Die WLB in Facebook}

\section{Hintergrund}

Beginnend mit den 90er Jahren des letzten Jahrhunderts hat das Internet eine so tiefe Verankerung und Verbreitung in der modernen Gesellschaft wie kaum ein anderes Medium vorher gefunden. Als selbstverständlich wird heutzutage erwartet, dass Institutionen sich mit ihren Informationen und Dienstleistungen im Internet präsentieren. Seit vielen Jahren ist die Website der WLB das Internetportal für die vielseitigen Angebote und Informationen der Bibliothek. Viele Angebote sind insbesondere auf die Nutzung im Internet ausgerichtet und erlauben dem Benutzer eine dauerhafte unbeschränkte Verfügbarkeit, die nicht an die Räumlichkeiten und Öffnungszeiten der Bibliothek gebunden ist.

\begin{abstract}
Die Website in der bisherigen Konzeption bietet weiterhin die klassisch lineare Kommunikation zwischen der Bibliothek und ihren Benutzern. Diese Art der Kommunikation bietet viele Vorteile und ist weiterhin die am stärksten nachgefragte Kontaktaufnahme mit der Bibliothek, um über das Internet Auskunft zu verschiedenen Fragestellungen und Anliegen in gezielter Weise zu erhalten. Die WLB bietet über das virtuelle Auskunftssystem Infodesk und E-MailAdressen viele Möglichkeiten, direkt mit den Mitarbeitern und den gewünschten Arbeitsbereichen in Kontakt zu kommen. Diese werden sehr stark genutzt, was sich auch in den jährlich steigenden Anfragen zeigt, die an die Information gerichtet werden. In allen Anfragen zeigt sich der Bedarf an Kommunikation mit der Bibliothek als Informationspartner.
\end{abstract}

